Linking a cytokine/protein biomarker signature with clinical outcome may help to identify and classify patient cohorts. Thus "intelligent" cytokine signatures can be used as biomarkers in human inflammatory diseases. There are however various risks associated with this approach; often it is impossible to obtain material from the site of inflammation and there are various often not well-known technical aspects crucial to obtain reliable and usable results. Although standardization has been prominent in day to day clinical practice, standardization of sample collection and laboratory assessments remains suboptimal. Inconsistency in sample collection can affect the results of biological assays and thus several characteristics require thorough evaluation and standardization. This standardization is not limited to assay validity and reproducibility but also pre-analytical treatment and appropriate specimen types.

Disclosure of Interest: None declared

DOI: 10.1136/annrheumdis-2017-eular.7203

\section{SP0068 CLINICAL INSIGHTS INTO JIA HETEROGENEITY}

\section{A. Consolaro on behalf of EPOCA Study Group. Istituto Giannina Gaslini, Genova, Italy}

Several epidemiologic surveys have documented a remarkable, yet unexplained, disparity in the prevalence of juvenile idiopathic arthritis (JIA) subtypes among different geographic areas or ethnic groups. Moreover, the therapeutic approach to JIA is not standardized and the availability of the novel and costly biologic medications is not uniform throughout the world. This disparity may have significant impact on disease outcome. The multinational study of the EPidemiology, treatment and Outcome of Childhood Arthritis (EPOCA study) is aimed to obtain information on the variability of JIA phenotypes in different geographic areas, the therapeutic approaches of pediatric rheumatologists practicing in diverse countries, and the disease status and outcome of children with JIA currently followed worldwide. Participation in the study was proposed to all pediatric rheumatology centers that are part of the Pediatric Rheumatology International Trials Organization (PRINTO), and to several centers in the US and Canada. Each center was asked to enroll 100 consecutive JIA patients or all consecutive patients seen within 6 months. Each patient received a retrospective and cross-sectional assessment. Parent- and child-reported outcomes were recorded through the administration of the Juvenile Arthritis Multidimensional Assessment Report (JAMAR). Participating countries were grouped into 6 geographic areas. Patients were then grouped according to their country's gross domestic product per capita (GDP) and the total expenditure on health per capita (HE) (source www.who.org). Currently, 8,325 patients from 44 countries have been entered in the web database. Comparison of main epidemiology, treatment, and outcome features across the different geographic areas was performed. Patients living in countries with GDP or HE below the median had lower frequency of remission, higher median cJADAS, higher frequency of damage, and were less frequently prescribed biologic DMARDs. These results were confirmed when analyses were conducted only in oligoarthritis or polyarthritis patients. These results provide further evidence of the wide difference of JIA characteristics across geographic areas in terms of age at disease onset, subtype prevalence, and frequency of anterior uveitis. Overall, patients living in non-Western countries had higher levels of disease activity and cumulative damage than patients followed in North America and Western Europe. This disparity in disease outcomes may be partially due to differences in the availability or affordability of biologics, as confirmed by the evidence of worst outcomes in countries with lower GDP or HE.

Disclosure of Interest: None declared

DOI: 10.1136/annrheumdis-2017-eular.7236

\section{THURSDAY, 15 JUNE 2017 \\ EULAR - EMA session}

\section{SP0069 REGISTRIES IN MUSCULOSKELETAL DISEASES AND THEIR REGULATORY USE}

R. Vesely. Rheumatology, Respiratory, Gastroenterology and Immunology Office, European Medicines Agency, London, United Kingdom

Patient registries collect information about individuals sharing health-related characteristics, for example, a particular disorder, a treatment or a procedure. While randomised controlled trials typically provide the primary evidence supporting marketing authorisations for new medicines, the patients studied may not be fully representative of everyone ultimately receiving the medicine and the trials may provide limited information about the natural history of the disorder. Information collected in patient registries is potentially of value for filling these evidence gaps in certain situations and for providing post-marketing safety and effectiveness information. Multiple stakeholders stand to benefit from using registry information in this way including patients, healthcare providers, policy makers, manufacturers and healthcare regulators.

In 2014, the EMA commenced a Registry Initiative aiming to optimise the use of registries in supporting medicines authorisations. Establishing a strategy of early engagement between marketing authorisation applicants and registry holders and a task force to support activities, a pilot phase was undertaken aiming to understand the barriers and enablers in using registries to support marketing authorisation applications and to inform the development of recommendations to optimise their use.

On 28 October 2016 the Agency organised Patient Registries Workshop to collect and discuss the information about experience from different patient registries in various therapeutic areas. The topics included:

- Benefits of registries for regulators

- Benefits of registries for HTA and payers

- Benefits for industry

- Benefits for clinicians and researchers

- Benefits for patients

- Challenges in collaboration between registries

- Technical challenges

- Governance

- Sustainability

Conclusions of the pilot and the workshop have been utilised in the following activities including workshops to support registries in individual diseases.

There are multiple advanced registries in RA and JIA. Utilisation of outcomes of these and other existing or newly planned registries in other musculoskeletal diseases for regulatory purposes current environment offers new opportunities that require further analyses and collaboration.

Disclosure of Interest: None declared

DOI: 10.1136/annrheumdis-2017-eular.7159

\section{SP0070 NEWS FROM OMERACT - IMAGING AND MORE}

\section{Strand. Stanford University School of Medicine, Portola Valley, United States}

Objective In rheumatoid arthritis (RA), MRI provides earlier detection of structural damage than radiography (X-ray) and more sensitive detection of intra-articular inflammation than clinical examination. This analysis was designed to evaluate the ability of early MRI findings to predict subsequent structural damage by X-ray. Methods Pooled data from four randomised controlled trials (RCTs) involving 1022 RA hands and wrists in early and established RA were analysed. X-rays were scored using van der Heijde-modified or Genant-modified Sharp methods. MRIs were scored using Outcome Measures in Rheumatology (OMERACT) RA MRI Score (RAMRIS). Data were analysed at the patient level using multivariable logistic regression and receiver operating characteristic curve analyses.

Results Progression of MRI erosion scores at Weeks 12 and 24 predicted progression of X-ray erosions at Weeks 24 and 52, with areas under the curve (AUCs) of 0.64 and 0.74 , respectively. 12-week and 24-week changes in MRI osteitis scores were similarly predictive of 24- week and 52-week X-ray erosion progressions; pooled AUCs were 0.78 and 0.77 , respectively. MRI changes in synovitis at Weeks 12 and 24 also predicted progression of X-ray joint damage (erosion and joint-spacenarrowing) at Weeks 24 and 52 (AUCs=0.72 and 0.65, respectively).

Conclusions Early changes in joint damage and inflammation detected with MRI predict changes in joint damage evident on subsequent $\mathrm{X}$-rays. These findings support the use of MRI as a valid method for monitoring structural damage in short-duration RCTs.

Disclosure of Interest: None declared

DOI: 10.1136/annrheumdis-2017-eular.7189

\section{THURSDAY, 15 JUNE 2017 Barrier free employment for young people with RMDs}

\section{SP0071 YOUNG PATIENTS: READY, BRILLIANT AND ABLE TO WORK! \\ L. Kullamaa. European Patients' Forum, Brussels, Belgium}

In May 2016, European Young Patients Group, an initiative representing young patients from the European Patients' Forum (EPF) and the European Multiple Sclerosis Platform (EMSP), organised a workshop in the framework of the European Youth Event (EYE) 2016 in Strasbourg that corresponded with one of the five main programme themes titled, Exclusion or Access: Crackdown on Youth Unemployment.

The physical and emotional symptoms of chronic conditions, together with social stigma and attitude, create significant barriers to young patients in the job market. With appropriate support, they, like all enthusiastic young people, can be assets for employers. Through interactive discussion, creative expression, education and open dialogue, the workshop aimed to challenge expectations and inaccurate perceptions about the abilities of young people with chronic conditions, tackle societal beliefs and stereotypes of individuals with chronic conditions, stimulate discussion to explore concrete solutions and develop practical actions for young people and their allies accessing employment and steer change to ensure young patients benefit from equal opportunities and treatment at work. By addressing these specific objectives, the workshop was to ultimately raise awareness of the extra burdens faced by young people with chronic conditions transitioning from education to employment, as well as bringing public attention to the stigma and discrimination that exists at both the recruitment stage and in relation to employees disclosing their health conditions. It also was to compliment and 
strengthen the EPF and EMSP's wider initiatives on tackling the multiple forms of discrimination faced by patients with chronic conditions.

The workshop demonstrated how many issues surrounding young people with chronic conditions transitioning into the labour market are still prevalent. The expectation of discrimination and stigma in the workplace, along with the unwillingness to disclose their condition when applying for a job for fear of rejection are confounded by young patients' experiences of negative employer attitudes. Moreover, the concept of quota systems and giving people with chronic conditions and/or disabilities preference among applicants for certain jobs possibly suggests that they are employed because they have a chronic condition and/or disability, not because of their abilities. This unambiguous form of positive discrimination also affects how young people manage information about their health. They feel forced to share about their condition to get an interview, however by doing so they risk being subjected to negative reactions in the interview or at the workplace. Young people with chronic conditions have a great deal to contribute to the workplace and more action should be taken to encourage and support employers with hiring them. Training and resources should be provided to managers and employees to raise awareness of the impact that chronic conditions can have on an individual, and how they can be assisted through small adjustments or specific care requirements. Employees should not assume what someone can or cannot do and how their condition affects them, therefore a place for an open discussion should be created. In addition, where quota systems apply, they should be re-evaluated and designed to recruit young patients on merit, in competition with other workers without a chronic condition and/or disability.

Disclosure of Interest: None declared

DOI: 10.1136/annrheumdis-2017-eular.7262

\section{SP0072 CHALLENGES AND POTENTIAL SOLUTIONS ABOUT STAYING IN WORK AS A YOUNG PERSON WITH AN RMD}

J. Andersen $1,2,3 .{ }^{1}$ Lupus Europe, Romford, Essex, United Kingdom; ${ }^{2}$ EULAR Young PARE, Zürich, Switzerland; ${ }^{3}$ Lupus Dk, Gigtforeningen, Copenhagen, Denmark

Introduction: A lot of young people with RMDs experience difficulties when it comes to staying in work after diagnosis. Some employers see a young person with an RMD-diagnosis as a liability. They are afraid it will cause a lot of difficulties with sick leaves and less work effort and in the end cost them a lot of money. Often the young person hasn't worked in the company for that many years (because of the age) and hasn't had the chance to make themselves indispensable/a valued work force yet, which makes it easy/obvious for the employer to terminate their employment.

The process of getting a diagnosis can often cause long periods of sick leave in the beginning and many employers don't understand that this will get better over time as the person gets used to having the disease and the medication has a chance to work properly.

Some young people choose not to tell their employer, that they have an illness at all, which can also be a big problem. When the employer and the work colleges don't know about the diagnosis, they cannot take special consideration to the person with the illness. Sometimes, you might a little extra break to be able to work an entire day and without it you risk wearing yourself down and having to quit the job yourself. If you choose to take the breaks yourself or perhaps fail at your job, because you try to do as much as the healthy persons, you risk getting fired, because nobody knows there is a good reason why you cannot do as much as you "should". Young and inexperienced persons can have difficulties demanding special treatment. They are often too insecure to stand their own ground and demand special consideration.

Objectives: I aim to examine the problems young people with RMDs face, when they want to stay in work and find potential solutions to these problems. How big is the problem? What can be done by the young people themselves? How can national patient organisations help them? How can EULAR PARE or other united European patient organisations help? Do we need new regulations? How do we make each country follow the EU-legislation?

Methods: I have asked young people with RMDs from all across Europe about their experiences with staying in work through social media and email. I have also asked patient organisations from all of Europe through email and personal meetings about their experiences, when it comes to young people with RMDs and work

Results: The challenges differ from country to country. In some countries having an RMD is seen as a taboo, a sign of weakness. In other countries, an RMDdiagnosis makes you no different than the rest of society and the expectations to what you can do are often too high. I will attempt to make a presentation of potential solutions to the different issues. How it could help, if patient organisations spread awareness about RMDs among employers all across Europe. How, if the young person told their employer, that they have an RMD, it might help them in the long run. There are several places where young people with RMDs can get help and advise on how to act, when they want to stay in work. There already exists EU-legislation on the area, but not all EU-states respect them and adhere to them - this has to be changed.

Disclosure of Interest: None declared

DOI: 10.1136/annrheumdis-2017-eular.7167

\section{SP0073 PRACTICAL EMPLOYABILITY SUPPORT FOR YOUNG PEOPLE WITH RMDS}

M. Mcallister. Working Well with Arthritis Joint Working Service, Arthritis Care, Glasgow, United Kingdom

Background: Arthritis Care is a UK national charity providing a range of support through three services;

Working Well with Arthritis - Joint Working employability service (Scotland only). $87 \%$ of Arthritis and Work Scotland 2016 survey respondents' state arthritis has a high or very high impact on work. Joint Working (JW) service aims to address this by:

Providing holistic employability support to individuals of working age to improve their capacity to manage their condition in work.

Providing employers support to increase understanding of arthritis within the workplace

Offering employers free Arthritis Awareness training

Young People and Families Service (YPFS) for age 10 - 25 years.

Series of free, one day or weekend workshops, to help reduce social isolation, increase confidence and management of condition.

Living Well with Arthritis offers adults access to self management support such as:

Managing pain/symptoms; activity/exercise; health walks, Tai chi for arthritis; peer support.

Objectives: Joint Working (JW) service aims to help individuals of working age with arthritis make informed decisions about work, support them to remain in, return to, or explore suitable work, training or alternative employability opportunities.

1 in every 1,000 young people in the UK has arthritis, YPFS complements medical treatment by providing additional social and emotional support, to help reduce isolation and increase resilience. With all services being delivered by Arthritis Care there is a natural crossover and support network for young people around transitioning from education to work.

The services aim to provide individuals with the knowledge, confidence, skills and support to empower them to make informed life and work decisions and access key support services - with the aim of improving their chances of remaining in work.

Method: There is a partnership and referral process to JW via NHS Rheumatology Occupational Therapy staff, within specific NHS areas, though referral to JW is open to anyone with arthritis.

Partnership working with; NHS, employability networks and Arthritis Care YPFS is key to the success of JW service.

Individuals with arthritis often find; communicating their needs, understanding their rights and navigating the system can be complex and overwhelming. Joint Working provides support through a range of mediums; 1 to 1 meetings (where practical), email and/or phone. Exploring areas of concern, improving understanding of rights, awareness of, and access to national employment support schemes, mainstream or disability employability services.

Results: Young people who have used Joint Working service have been supported to access a range of services; obtaining specialist equipment or travel support to remain in work, returning to work after many years unemployed, work placement to support university course, volunteering or specialist employability opportunities, Conclusions: The employability interventions have positive psychosocial benefits, reducing feelings of isolation, increasing confidence and raising aspirations.

People with arthritis value the opportunity to discuss any issues they have remaining in, returning to or embarking on a career path.

The ongoing access to all Arthritis Cares services, ensure young people have a long-term support network.

Maureenm@arthritiscare.org.uk

Disclosure of Interest: None declared

DOI: 10.1136/annrheumdis-2017-eular.7149

\section{THURSDAY, 15 JUNE 2017 \\ Epidemiology of rheumatic and musculoskeletal diseases - a critical appraisal}

\section{SP0074 THE FUTURE OF EPIDEMIOLOGICAL RESEARCH IN RHEUMATIC AND MUSCULOSKELETAL DISORDERS}

L. Gossec. Rheumatology Department, Paris 06 University, Pitié-Salpétrière Hospital, Paris, France

Epidemiological research has gone through a very exciting time over the past years in rheumatic and musculoskeletal disorders (RMDs). The future is just as exciting. Although 'traditional' epidemiology in terms of prevalence and incidence of diseases is quite well-known now, there are many gaps to our knowledge in particular in the field of outcomes research (what to assess, when and how?) but also in the field of predictive factors (e.g. predictive factors of severity and of treatment response). Better knowledge in outcomes assessment is key to feasibility of standardised assessments and is the basis of shared decision making. Better knowledge of predictive factors will open the door to truly personalised medicine. Some future directions will be discussed in this talk. 